\title{
Assessment of wind power predictability as a decision factor in the investment phase of wind farms
}

\author{
R. Girard, K. Laquaine and G. Kariniotakis \\ MINES ParisTech, Center for Energy \& Processes, Sophia Antipolis, France.
}

\begin{abstract}
The ability to predict wind power production over the next few hours to days is prerequisites for the secure and economic operation of power systems with high wind power penetration. From the point of view of a producer participating in the day-ahead electricity market, lack of predictability at a wind power production site results in imbalance costs. This paper aims at quantifying the impact on market revenue of, respectively, the predictability and the capacity factor of a wind farm or a cluster of wind farms. This is done through a real-life case study in West Denmark, including wind farm production data and market data. Finally, we make a prospective analysis under the assumption that the imbalance price settlement mechanism will remain the same.
\end{abstract}

Keywords: Wind power resource assessment, Wind power predictability, Market design, Planning policy, Sensitivity analysis, Investment problem

\section{Introduction}

The share of renewable energy sources in the energy mix of several countries worldwide is rapidly increasing. As regards the European Union (EU27), the European Commission has set a target of $20 \%$ of EU-27 energy consumption to come from renewable sources. Wind energy is anticipated to be a major contributor to this target with an installed capacity that is expected to extend from 74.7 GW by end of 2010 in EU-27, to 230 GW by 2020 according to EWEA projections (see [1]).

Such large-scale integration of wind energy raises several challenges in operating and managing power systems. It is now recognized that accurate short-term forecasts of wind farms' power output over the next few hours to 
days are important factors for the secure and economic operation of power systems with high wind power penetration [2]. The future evolution of installed wind capacity entails building a significant number of new wind farms across Europe. As most windy sites are already occupied in certain countries (e.g. in Spain [3] and Germany [4]), wind farm developers will have to deal with more and more complex terrains in the future. Previous works, like the benchmarking exercise performed in [5] have shown to what extent predictability is dependent on terrain complexity; the higher the complexity, the lower the predictability. It was shown also in [6] that predictability tends to decrease when wind speeds increase. In such a case, a site's predictability is denoted by the level of accuracy of short-term wind power predictions that can be obtained for that site.

Today, significant R\&D efforts are being undertaken to improve the performance of wind power prediction models and related weather forecast models. Increased overall wind power predictability is expected to be beneficial for several actors, such as transmission or distribution system operators, for efficiently performing functions such as estimating reserves, unit commitment, congestion management, etc. Independent power producers could benefit from increased predictability when they participate in an electricity market.

Since it is not possible to imagine a power system with unpredictable generating means, it is difficult to quantify the economic benefit of increasing predictability. A direct consequence of this is the difficulty in devising clear economic incentives aiming at greater predictability. However, when participating in the day-ahead market, deviations of the produced energy from the contracted energy (imbalance) due to forecast errors are exchanged at a different price called the imbalance price, resulting in imbalance costs from the wind power producer's point of view. This direct translation of wind power forecast errors into a financial cost, as well as strategies for the reduction of this cost, have already been studied (see e.g. in $[7,8,9,10]$ ).

The investment decision on the placement of wind turbines has been analyzed in previous works. Ref. [11] investigates to what extent optimal allocation of wind farms in Germany can reduce wind power fluctuations through aggregation while [12] proposes a model in the frequency domain to assess the power reduction induced by cut off in high wind conditions. These works have shown that the aggregation of wind farms can produce significant effects in terms of variability and cost reductions.

The aim of this paper is to quantitatively assess the role of predictability 
when expanding a wind farm portfolio, with real market data and real wind farm production data. Here predictability of a new wind farm is supposed to be determined by its location and by the aggregation effect with the rest of the portfolio. The actors concerned could be independent power producers, wind farm developers, aggregators or virtual power plant operators that need to decide where to install a new wind farm, or how to compose an optimal portfolio of wind farms for participating in an electricity market. In addition, penalties paid by producers that deviate from the day-ahead contract are settled by the transmission system operator and market operator, who will thus be concerned by the results of this paper.

These questions relate to a case where the revenue of a wind farm as an investment is generated by the wind farm's direct participation in an electricity market and not through a subsidy-based scheme, i.e. a feed-in tariff system. A large share of Europe's current installed capacity is supported by feed-in tariff schemes. However, as penetration increases, Member States are tending to switch to direct market participation mechanisms for wind farms. This means that a high share of wind farms will be developed under such mechanisms in the future.

In a subsidy-based supporting scheme, decisions on where to install a new wind farm are taken based on well-established "resource assessment" practices. Given the fixed remuneration per $\mathrm{kWh}$, predictability does not play any role in decision-making. In contrast, when wind farms participate directly in an electricity market, wind farm revenue is generated from the market and is affected by the short-term predictability of wind farm production. Indeed, imbalance prices are applied to imbalances.

As mentioned above, well-established methodologies exist in the case where a decision has to be made based solely on a site's wind potential. In other words, the site with the highest potential tends to be chosen. However, this is not the case in the new electricity market context. This paper contributes to this tendency, by studying the new questions that are increasingly being posed by end-users: Can a compromise between resource potential and predictability be beneficial when choosing between two sites to install a wind farm?

When a wind farm participates in an electricity market, the revenue is a function of the contracted energy, the spot prices, the difference between the contracted energy and the produced energy and the imbalance prices. The induced imbalance costs reduce the revenue proportionally to forecast errors. One might then ask the question that, if some compromise has to be found 
when choosing between two sites, let us say one with high potential but low predictability (i.e. a complex terrain site) and one with lower potential but higher predictability (i.e. a flat terrain site), then such a compromise might lead to choosing the site with lower potential if the loss in revenue can be compensated by lower penalties. Taking this reasoning one step further, one might study how to optimally extend a portfolio of wind farms by adding new wind farms so that the ensemble has an optimal performance in the market.

Predictability here is seen from the producer's point of view. It is however a very important aspect as such for the power system and market operators, who may want to incite wind farm operators to adopt practices that increase predictability so that wind production is the source of less imbalances. The assessment in this work may contribute to defining incentives in this direction.

In this paper we propose a methodology to study the above questions, which is presented in Section 2 together with the test case of several hundred wind farms in Denmark and the market assumptions that we used. In Section 3 this methodology is applied to evaluate the importance of predictability versus that of the capacity factor on the revenue generated by a wind farm. Two cases are considered, one where a producer considers the installation of a single wind farm, and the second where a portfolio or aggregation of wind farms is extended. Before the conclusions, Section 4 proposes a prospective discussion of the results.

\section{Methodology and Description of the case study}

\subsection{Methodology overview}

In our approach we consider the current performance level of state-ofthe-art forecasting technology as being given. A reasonable range of possible levels of predictability is considered in the case study. Better levels of predictability may be achieved in the future through further research. This could include more collaboration with the meteorological community on how to better use meteorological models, elaboration of more accurate power prediction methods especially for extremes, and improved processes for checking data quality, a.o.

Here, however, we attempt to increase given levels of predictability through aggregating wind farms, which can be considered as a form of physical hedging. Previous works have studied the spatial smoothing effect that can be achieved in a wide area and can result in a substantial increase in the predictability of the aggregation compared to individual wind farms (see e.g. 
[13][14]). Other techniques for physical hedging could be based on coordinating wind farms with either storage devices or conventional generation physical hedging technique [8].

The methodology can be broken down into two steps. In the first step we simulate individual wind power producers' participation in the day-ahead electricity market. This results in quantified revenues per producer. In the second step we make a statistical analysis of the revenue, capacity factor (CF, produced MWh per year / $(8760 \mathrm{~h} \mathrm{x} \mathrm{Pn}))$ and predictability at a wind farm (expressed by the NMAE defined in Subsection 2.4). This allows us to evaluate the relative importance of predictability w.r.t. the capacity factor for increasing revenue. This methodology is applied twice

1. initially only with wind farms participating in a market independently;

2. then allowing clusters of wind farms of up to 16 in size to jointly participate in the market. This makes it possible to study the aggregation effect.

We consider realistic time series of wind power production and associated prediction errors through a real-life test case. These errors are synchronous to the market data so that any correlation remains present in both types of data. The methodology for obtaining these errors is described in Subsection 2.4. In order to compute the revenues of a wind power producer participating in the day-ahead market, we use a day-ahead market model in Subsection 2.3. Real-world time series of market spot and imbalance prices from NordPool are considered.

The results of the first step (i.e. revenue per year per wind farm in the test case) are analyzed in Section 3 with appropriate statistical tools including parametric regression and a general form of variance analysis. We estimate quantitatively how the revenue of a wind farm (or a portfolio of wind farms) relates to the $\mathrm{CF}$ and the predictability. This is done through either a parametric or a non-parametric regression over all producers of the test case. This relation is used to carry out a sensitivity analysis that allows us to compare the value of the capacity factor with that of predictability to determine the revenue. The sensitivity analysis proposed here is inspired by the global sensitivity analysis (see e.g. [15]) and relies on the empirical computation of the share of the revenue's variance that is explained by the capacity factor, (respectively by the NMAE). 


\subsection{The case-study}

This paper builds upon a case study in Western Denmark. This area is characterized by high wind potential and flat landscapes. It was chosen due to the high number of wind farms located there and the existence of sufficiently long tracks of data (4 years) to permit us to produce results with a statistical meaning.

The available wind power production data of neighboring wind farms within the considered area are grouped according to the nearest transformation station. Among the 565 transformation stations present in the area, 361 were discarded due to poor data availability or because of a sharp change in the installed capacity of the related wind farms during the considered period. 22 additional stations were discarded because of significantly low installed wind capacity (below $1 \mathrm{MW}$ ). The remaining 182 transformation stations still form a substantial dataset used in this paper and represented on the left-hand side of Figure 1 with the associated capacity factor. Hereafter, since the data measured at a given station is the sum of wind turbine measurements, we will talk of wind farms rather than transformation stations. The data cover the period 01/2006-12/2010 with an hourly resolution.
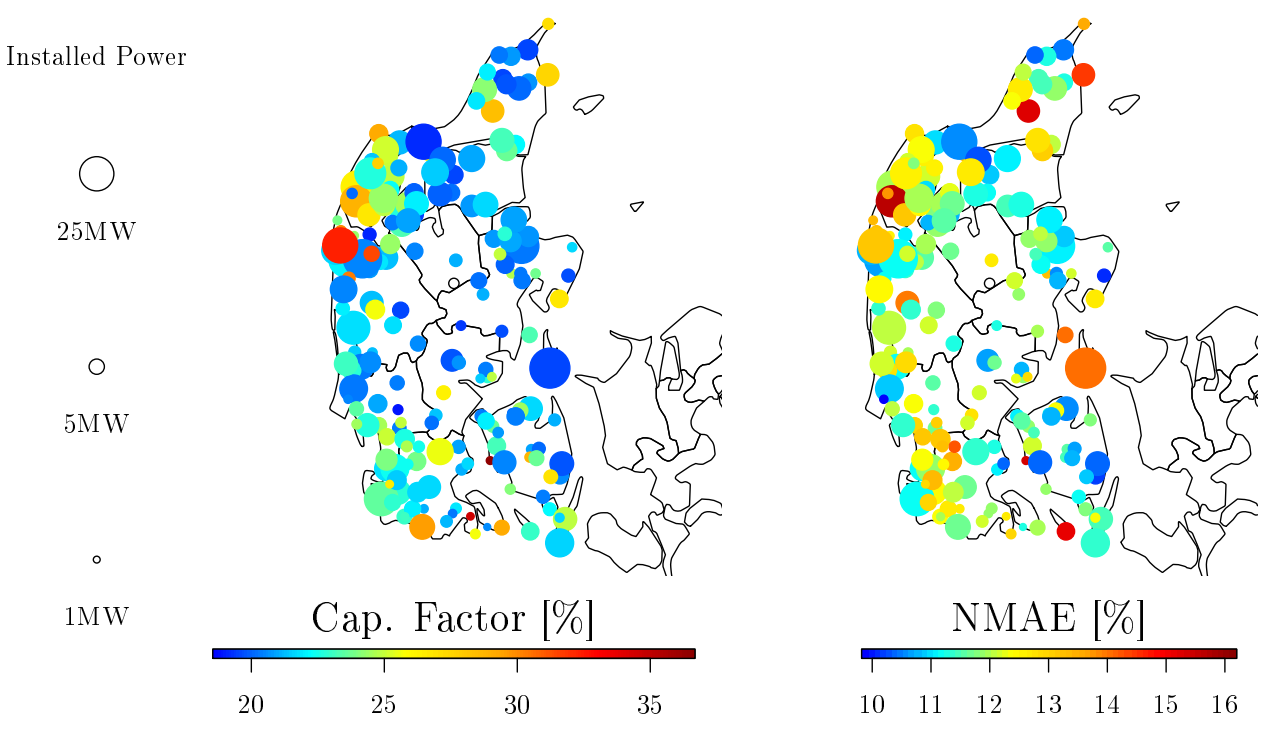

Figure 1: Location of the transformation stations analyzed in this work. Circle size represents installed wind farm capacity. The color code gives the day-ahead NMAE [\% of Nominal Power] as defined by Equation 4. 


\subsection{Day-ahead market model}

Power producers operate daily in electricity markets. In day-ahead markets, producers submit their quantity-price bids the day before delivery, and a single market price is defined by matching the aggregated offer and demand curves. E.g. in the NordPool Elspot day-ahead market, bids must be settled before noon for every hour of the following day.

When trading their production on the market, wind power producers, as well as conventional power producers, are economically responsible for any imbalance between contracted and delivered energy. Market participants are said to be balance-responsible parties. To avoid regulation costs, wind power producers have to make suitable decisions about their energy contracts before delivery, despite being uncertain as to future wind speed intensity and thus their wind power production. In other words, the limited predictability of wind generation makes it particularly sensitive to regulation costs. These costs translate the wish of the Transmission System Operator (TSO) to provide power producers with incentives for minimizing imbalances so that the equilibrium between generation and load can be maintained at all times and the system kept stable.

In order to simulate wind energy producers' participation in an electricity pool, we use the market model of [7]. The main assumptions of this model are that wind power producers are considered as price-takers, their bidding policy cannot impact imbalance prices, and they act in electricity markets as conventional producers that do not benefit from derogatory rules.

Following the notations introduced in [7], $E_{t+h}^{c}$ denotes the level of contracted energy for time $t+h$ (we restrict the study to hourly resolution, $t$ is the bidding time and $h$ is a number of hours). $R_{t+h}$ denotes the revenue at look-ahead time $t+h$ of a market participant bidding at time $t$ an amount of energy $E_{t+h}^{c}$ but actually generating $E_{t+h}^{*}$. This revenue can be formulated as the combination of the income from selling the actual wind generation at the spot price $\pi_{t+h}^{c}$ minus the imbalance cost:

$$
R_{t+h}=\pi_{t+h}^{c} \cdot E_{t+h}^{*}-T_{t+h}^{c}, \forall h=13, \ldots, 36 .
$$

The imbalance is defined as $d_{t+h}^{*}=E_{t+h}^{*}-E_{t+h}^{c}$ and consequently, the imbalance cost $T_{t+h}^{c}$ is given by

$$
T_{t+h}^{c}= \begin{cases}\pi_{t+h}^{c,+} \cdot d_{t+h}^{*}, & \mathrm{~d}_{t+h}^{*} \geq 0 \\ -\pi_{t+h}^{c,-} \cdot d_{t+h}^{*}, & \mathrm{~d}_{t+h}^{*}<0\end{cases}
$$


with $\pi_{t+h}^{c,+}$ and $\pi_{t+h}^{c,-}$ the imbalance prices for positive and negative imbalances respectively. Without any temporal precision, revenue stands for the cumulative revenue of a producer participating in the electricity market for the whole period considered; since our case study has been elaborated over more than one year, we express revenues and imbalances in euro per installed MW per year.

Finally, the market participant is assumed to bid the amount of predicted energy for each hour. The quantity-price bid is assumed to be accepted and is then equal to the contracted energy $E_{t+h}^{c}$, which is determined by the power forecast $\hat{P}_{t+h \mid t}$ multiplied by the time step $\Delta \tau$ at look-ahead time $t+h$ :

$$
E_{t+h}^{c}=\hat{P}_{t+h \mid t} \times \Delta \tau, \hat{P}_{t+h \mid t} \in\left[0, P_{n o m}\right], \forall h=13, \ldots, 36
$$

In our case study, power producers operate under the NordPool Power Exchange rules. This market gathers all power producers from the Nordic Countries and is divided into several areas. Western Denmark corresponds to the DK-1 territorial subdivision of NordPool Power Exchange. The market participation simulation is conducted according to this market's specific rules. In particular, power producers are not charged with penalties if their energy imbalance goes against market regulations, also referred to as a dual pricing mechanism. Typically, a power producer operating in this market, and currently producing less than contracted, will have their energy recompensed at the spot price if market is down-regulating, that is to say, if the total amount of energy generated among market participants is greater than consumption.

\subsection{Wind power forecasts}

For the purpose of this study, wind power production forecasts $\hat{P}_{t+h \mid t}$ issued at time $t$ for look-ahead time $t+h$ were derived using numerical weather

predictions of wind speed and direction at $100 \mathrm{~m}$, denoted as $\hat{W} S_{t+h \mid t}, W D_{t+h \mid t}$ respectively, provided by the Danish Meteorological Institute, and hourly power production measurements from the past two hours denoted as $P_{t}, P_{t-1}$ :

$$
\hat{P}_{t+h \mid t}=\hat{f}_{h}\left(\hat{W} S_{t+h \mid t}, \hat{W} D_{t+h \mid t}, P_{t}, P_{t-1}\right) .
$$

For a given horizon $h$, the regression function $\hat{f}_{h}$ is obtained with Random Forest, a non-parametric estimation procedure, implemented in the RandomForest [16] package of statistical analysis software R [17]. The parameters of 
the model are estimated using the first 24 months of the data. The remaining period of $T=682$ days is used as a testing set.

For any wind farm at position $x \in \mathcal{P}(\mathcal{P}$ is the set of all 187 considered wind farm positions), the corresponding normalized forecasting error in the testing set is

$$
\operatorname{Err}_{t+h \mid t}^{x}=\left(P_{t+h}^{x}-\hat{P}_{t+h \mid t}^{x}\right) / P_{n o m}^{x} \times 100[\%]
$$

where $P_{n o m}^{x}$ is the nominal capacity of the wind farm. In the testing set, the normalized average mean absolute error for the day ahead is defined by:

$$
\mathbf{N M A E}^{x}=\frac{1}{T} \sum_{t=1}^{T} N M A E_{t}^{x} \text {, where } N M A E_{t}^{x}=\frac{1}{24} \sum_{h=13}^{H=36}\left|\operatorname{Err}_{t+h \mid t}^{x}\right| \text {. }
$$

The values of $\left(\mathbf{N M A E}^{x}\right)_{x \in \mathcal{P}}$ are shown by Figure 1 with a color code.

In Equation 4, the errors for look-ahead times $t+13 h$ up to $t+36 h$ are considered to account for the window in the predictions that correspond to the day-ahead market. Predictions are considered to be made at $12 \mathrm{~h} 00$ with the available data up to that point. The values of $\left(\mathbf{N M A E ^ { x }}\right)_{x}$ per wind farm are depicted in Figure 1 with a color code.

\section{Consideration of predictability as a design parameter}

\subsection{Introduction}

In this section, we apply the second step of the methodology presented in Subsection 2.1 and analyze the results obtained. More precisely, the revenue obtained from a wind farm or a portfolio of wind farms is derived from hourly revenues defined by Equation 1. From this equation, it is expected that this revenue will be approached by a function $f$ of the average production of the wind farm during the considered period and a measure of the average forecast error. The average production is expressed relatively to the installed MW with the Capacity Factor $(\mathrm{CF})$, similarly the average error is expressed by the NMAE defined by Equation 4.

We use our case study to show that such a function $f(\mathrm{CF}, \mathrm{NMAE})$ exists and that it enables us to obtain the revenue with high accuracy. We use variance analysis to measure the strength of the contribution of $\mathrm{CF}$ and NMAE to revenue. In our case, the share of variance in a variable $Y$ that is explained 
by a variable $X$ is relative to a regression model $Y=\hat{g}(X)+\epsilon$ and estimated by the coefficient of determination $R^{2}=\operatorname{var}(\hat{g}(X)) / \operatorname{var}(Y)$ of this model. Note that measures of error other than the NMAE have been considered (such as root means square error or asymmetric error measures), but that none of them improves the quality of $f$ or changes the results significantly. We used the day-ahead wind power forecasts presented in Subsection 2.4, and the real Nordpool market data (energy prices, positive imbalance prices, and negative imbalance prices) for the 682 days of the considered period. In Subsection 3.2 we consider a case where each wind farm participates individually in the electricity market, and for each wind farm the result of the simulation is a revenue (in Eur / installed $M W /$ year $[$ Eur $/ M W /$ year $]$ ) and a total imbalance cost (in $[E u r / M W /$ year $]$ ). In Subsection 3.3 we obtain results of a similar nature, but where groups of wind farms are allowed to be considered as a single market participant (instead of single wind farms only).

\subsection{Effect of predictability and capacity factor at a single wind farm}

The predictability of a single wind farm is directly connected to the imbalance penalties paid, since greater errors lead to higher penalties. Initially, market participation is simulated individually for each of the wind farms considered. The capacity factor as well as the revenue and the imbalance penalties (both defined in Subsection 2.3) induced during the testing period are estimated. In the first scatter plot in Figure 2, we can see that the relationship between imbalance costs and overall predictability per wind farm, as expressed by the NMAE, is linear with a good coefficient of determination.

Concerning revenue, the upper-right graph of Figure 2 shows that it is very well explained by the wind farms' capacity factor. This implies in particular that in the current market context, there remains a very slight possibility of increasing the revenue with any parameter other than the capacity factor. Furthermore, the lower left graph in the same Figure shows that the NMAE has a significant positive correlation with the capacity factor, and since larger capacity factors produce larger errors, it is clear that on average greater errors correspond to higher revenue. This positive correlation is relatively well explained by the fact that a large capacity factor means higher production, and higher production often shows higher variability and lower predictability. The NMAE values obtained for the wind farms considered range from $[9.5 \% ; 16 \%]$. Despite the flat terrain, this range is not narrow but includes typical values that can be obtained for wind farms on flat or more complex terrains for the low and high NMAE values of the range respectively. This 


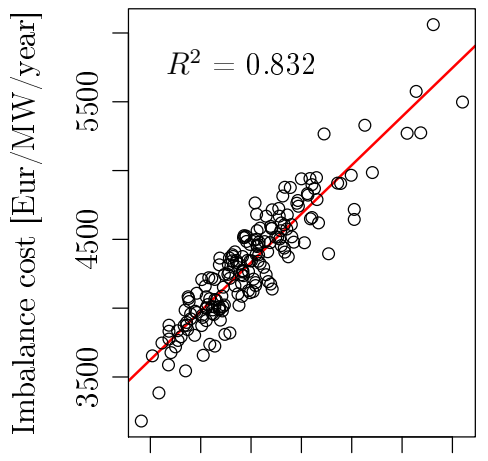

$\begin{array}{lllllll}10 & 11 & 12 & 13 & 14 & 15 & 16\end{array}$

NMAE [\% Nom. Pow.]

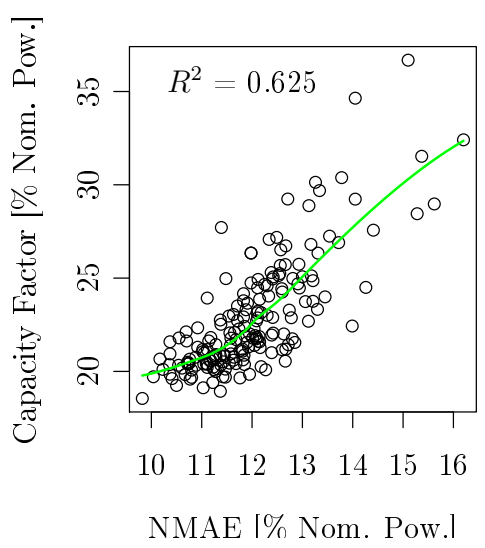

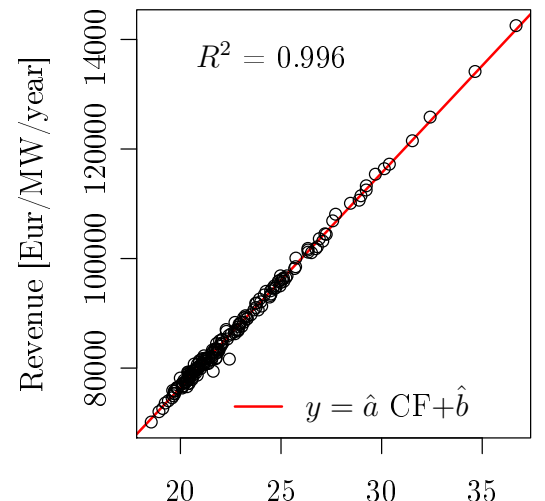

Capacity Factor $[\%$ Nom. Pow.]

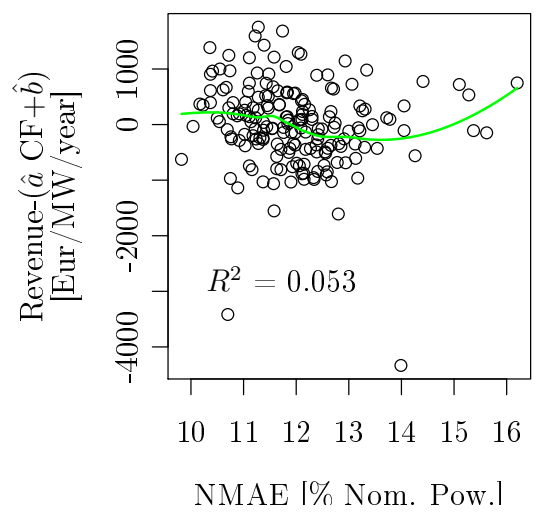

Figure 2: Relations between capacity factor, imbalance cost, revenue and predictability. Red lines are obtained through linear regression (the corresponding coefficient of determination is given). The green line is obtained with a local polynomial fitting (associated $R^{2}$ is given)

creates a counter argument to the fact that this test case could reflect typical predictability for flat terrains.

Coming back to the results of Figure 2, a typical situation can be derived. If one had to choose between two wind farms, one (say farm A) with $\mathrm{NMAE}=11 \%$ and another (say farm B) with $\mathrm{NMAE}=13 \%$, the imbalance cost saved could be around $0.85 \mathrm{kEur} / \mathrm{MW} /$ year (see the top-left graph of Figure 2). However, it is likely that the difference in predictability will translate into a difference in the capacity factor, in our typical example of farm A and farm B, the bottom left graph of Figure 2 shows the unlikelihood of 
having comparable capacity factors and on average this difference is around 4 points of capacity factor, which can represent $20 \mathrm{kEur} / \mathrm{MW} /$ year in revenue (according to the upper-left graph of Figure 2). The difference in the two quantities (0.85 and $20 \mathrm{kEur} / \mathrm{MW} /$ year) is large enough for us to conclude that the capacity factor will dominate in the decision-making process. The upper-right graph of Figure 2 shows the reliable linear relation that exists between the revenue and the capacity factor, the residuals of this relation are shown as a function of predictability to the bottom right of the Figure. Note that there is a difference between considering predictability in the resource assessment phase and considering predictability for a given installed wind farm. In the second case, not treated in this paper, the capacity factor is fixed. In this case the relation between predictability and imbalance cost, shown in the upper-left graph of Figure 2, gives the typical financial benefit that can be obtained.

As a conclusion to this analysis, the revenue that can be obtained from a single wind farm participating in the electricity market can be approached by a linear function of the capacity factor. The residuals of this approximation have a variance of $0.4 \%$ of the revenue's variance and only $5.3 \%$ of this residual variance can be attributed to NMAE. This means that NMAE impacts around $0.02 \%$ of the revenue's variance, and shows the poor value of predictability when used as a design parameter for a single wind farm participating in the electricity market.

\subsection{Effect of wind farms aggregation}

An efficient approach to increasing predictability is through aggregating wind farms. Aggregation allows forecast errors to compensate each other. This effect is more pronounced since the correlation between the wind farms' production decreases as their distance increases. At the scale of Denmark, spatial correlation is still relatively high but the large number of wind farms makes it possible to explore the effect of aggregation in detail. It is clear that a higher spatial smoothing effect could be expected for a larger country or for Europe (see e.g. [14]).

In contrast to the previous Subsection in which we examined the role of predictability when deciding on installing a single wind farm, here we examine a case in which a wind power producer, or an aggregator, is willing to install a group of wind farms. Based on the same case study as the preceding subsection, we considered aggregated wind farms instead of single wind farms. Since the potential number of combinations is too high to be 
considered, we reduced the analysis to a random set of combinations of a size not greater than 16 wind farms. The number of combinations generated is 65535. Each combination of wind farms is then considered as a new wind farm and the analysis produced in the preceding section is reproduced.
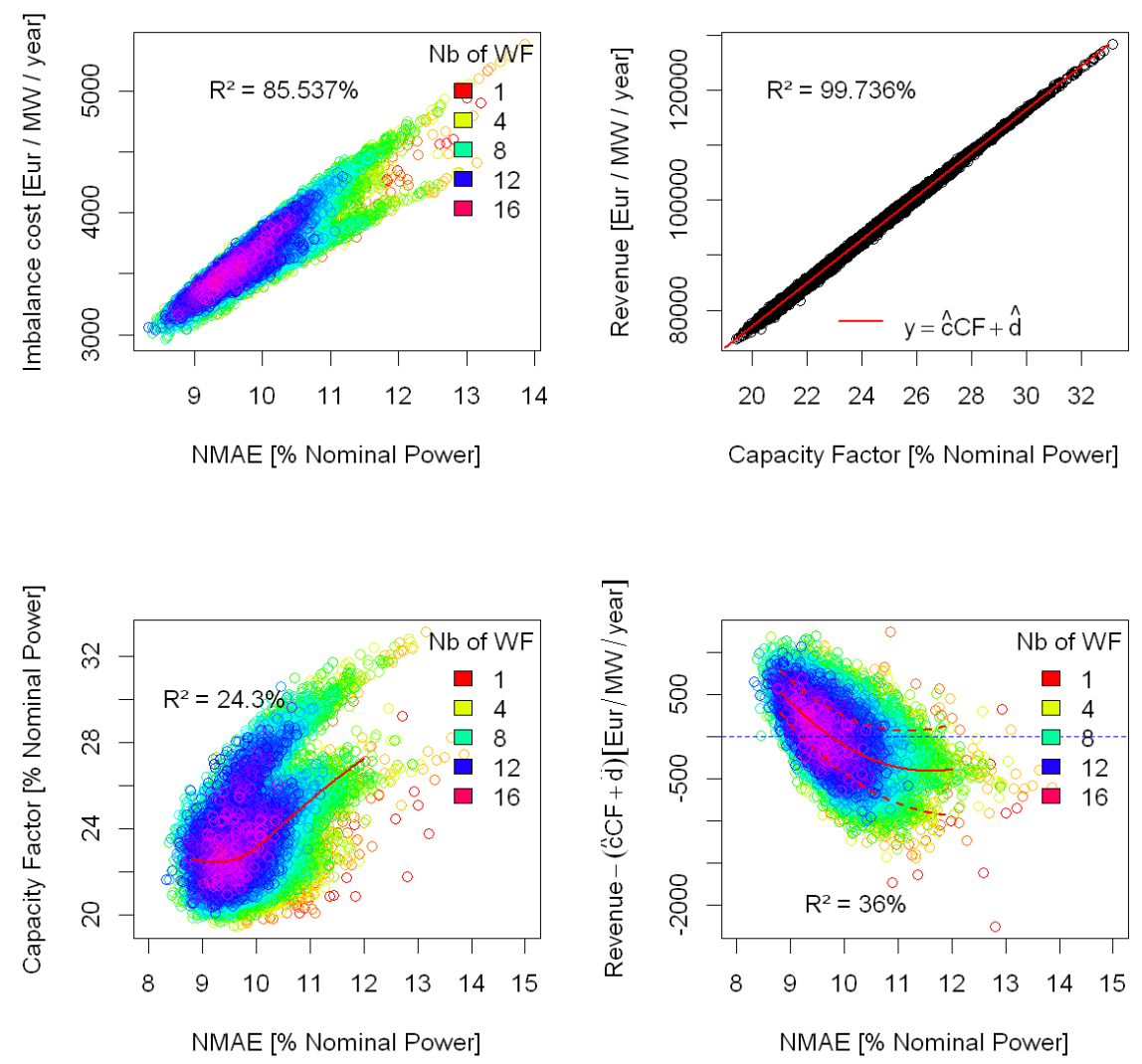

Figure 3: Relations between capacity factor, imbalance cost, revenue, predictability, and the number of wind farms in the portfolio. The solid red lines in the two upper graphs are obtained through linear regression (the corresponding determination coefficients are given). For the bottom-left plot, it is obtained using local polynomial fitting, while for the bottom-right plot it is obtained through second-order polynomial estimation (associated $R^{2}$ is given). The red dashed lines on the right give the conditional quantiles (90\% upper and $10 \%$ lower) obtained with second order polynomial models. The color code gives the number of wind farms within a given cluster.

In Figure 3 we present the results obtained. Each point in the plots repre- 
sents a combination of wind farms. Unsurprisingly, the aggregation does not change the relation between capacity factor and total revenue, as indicated by the coefficient of determination which remains the same. The lower-right plot presents the relationship between the residual revenue, which is the difference between the actual revenue and the revenue estimated by a linear function of the capacity factor. The link between the residual revenue and the NMAE, measured by the coefficient of determination, is much stronger in the case of combinations $(36.1 \%)$ than in the case where only single wind farms are considered (5.9\%). The strength of this relationship increases in line with the number of aggregated wind farms, but at the same time the spread of the NMAE values along the portfolio decreases with the size of the portfolio (i.e. number of wind farms in the combination). This means that the NMAE impacts around $0.15 \%$ of the revenue's variance.

Let us now analyze this relationship in more detail. For the whole range of possible NMAE values, the fit of the lower right-hand graph in Figure 3 is obtained with a local polynomial. If restricted to the range of NMAE between $8.8 \%$ and $12 \%$, a second order polynomial allows us to obtain the same fit, which means that, in this case, the revenue in Euro per installed MW per year for a group of wind farms is determined by the following equation:

$$
\forall \mathrm{NMAE} \in[8.8,12], \mathrm{CF} \in[19,39], \quad \mathrm{Rev}=A(\mathrm{CF})+B(\mathrm{NMAE})+\epsilon
$$

With

$$
\begin{array}{cc}
A= & -2173+3960 \times \mathrm{CF}, \\
B= & -448.25 *(\mathrm{NMAE}-9.753)+121.7 \times(\mathrm{NMAE}-9.753)^{2}
\end{array}
$$

where Rev is the annual revenue in $[E u r / M W / y e a r], C F$ is the capacity factor in [\% Nom.], NMAE the normalized mean absolute error in [\% Nom.] (as defined by Equation 4) and with $\epsilon$ a residual term. For NMAE ranging out of $[8.8,12]$ there are not enough data in our test case for us to be sufficiently confident in the second order polynomial model or other model. The relative importance of each term in Equation 5 can be quantified with the estimation of their standard deviations and average values as done in Table3.3.

The relative difference of magnitude in the variance (squared standard deviation) of $B, A$ and the revenue show the relatively small added value of predictability with respect to the capacity factor when trying to quantify the 


\begin{tabular}{|c|cccc|}
\hline Term in Equation 5 & Rev & $A$ & $B$ & $\epsilon$ \\
\hline \hline Standard Dev [€/ MW /year] & 6318.1 & 6309.4 & 194.92 & 259.84 \\
\hline \hline Average [€/ MW / year] & 89162.7 & 89157.0 & 0 & 0 \\
\hline
\end{tabular}

revenue. However, Equation 5 allows us to quantify the joint effect of capacity factor and predictability in the case when aggregation is allowed and, even if the effect is small, using this relation can lead to expected improvements in revenue. For example if two wind farms $X_{1}, X_{2}$ are expected to have a capacity factor of $25 \%$ and $24.9 \%$ respectively, then in the case where wind farm $X_{1}$ has predictability $N M A E^{1}=11 \%$, wind farm $X_{2}$ has to reach predictability of better than $N M A E^{2}=9.69 \%$ to have an expected revenue higher than wind farm $X_{1}$. In the case when $N M A E^{1}=10 \%$, then the wind farm 2 has to reach $N M A E^{2}=9.18 \%$. In addition, if the increase in revenue obtained by a predictability increase in this case allows us to compensate the loss caused by the difference in capacity factor, the financial gain is only twice as high as the standard deviation of $\epsilon$ in Equation 5.

If the two wind farms considered now have capacity factors of $24 \%$ and $25 \%$, with wind farm $X_{1}$ having predictability $N M A E^{1}=11 \%$, then wind farm 2 has to reach $N M A E^{2}=5.67 \%$ (if we admit that Equation 5 is for $N M A E<8.8 \%$ ). To our knowledge, such a difference in NMAE with a fixed prediction model cannot be encountered. As a conclusion, the aggregation effect makes it possible to increase the strength of the impact of predictability on the revenue. However, we have shown that in any reasonable case, this strength is still not comparable to the impact of the capacity factor. In other words, it is clear from the above results that it cannot be of benefit for an independent wind power producer participating in the day-ahead electricity market to consider predictability as a primary selection parameter for wind farm location or for composing aggregation portfolios.

\section{Prospective discussion of the results}

The results and conclusions of the preceding section were obtained with real-life data from the years 2008 - 2011 including production data, forecast data, market prices (spot and imbalance). There are two issues that one has to consider about the generalisation capacity of the applied in-sample analysis to consider the whole life-time of a wind farm (i.e. 20-25 years) .

The first one is that the exact capacity factor is not known in advance 
for a wind farm that is going to be installed and 1 year of met mast data is often used to its estimation. This common practice is known to result into an average error lower than 10\% (see e.g. [21] ). Similarly predictability cannot be estimated precisely before the wind farm installation. The consequence in practice is that the residual term in 5 might be larger, which would in turn reduce even more the impact of predictability.

The second issue is related to the prices of electricity. Indeed, if the 20 year average wind speed and predictability are not likely to change in the future (except maybe in the case of a major climate change) the situation about the spot price of electricity and the imbalance cost is more complex. In the current situation, the impact of errors on revenue is settled by Equation 1. If we assume that the use of this equation will be pursued in the future, it is clear that the impact of predictability on revenue will only increase in comparison with the impact of the capacity factor if the ratio between the imbalance price and spot prices increases. For this reason, in Subsection 4.1, we explore the significant relationship between spot price, imbalance prices and aggregation of errors in the NordPool market. In Subsection 4.2, assuming that the logic behind this relationship will remain in the future, we draw conclusions about the value of predictability as a design parameter in the future.

Finally, it is worth noticing that the use of advanced bidding strategies,such as an asymmetric cost function in the bidding, or the use of a physical hedging mean, such as storage, that would increase predictability without being related to the localisation of the wind farm, would further reduce the role of predictability in the resource assessment phase. In the second case however, one has to introduce the cost of the storage solution in the economic calculation.

\subsection{The imbalance price equation}

For the Danish system and the Nordpool market, a strong relationship exists between spot price, imbalance prices and aggregation of wind power

forecast errors. This relationship, illustrated by the 3 graphs of Figure 4 reflects the logic of forming imbalance prices whereby the cost of forecast errors for the transmission system operator is shared between wind power producers. Indeed, this cost is closely related to the total energy forecast error times, the spot price and Figure 4 shows that this last quantity is often almost equal to the sum of imbalance costs over all market participants. This is not far from the predicted relationship with imbalance prices. 
Note that the exact mechanism allowing us to compute imbalance prices is more complex, as is the market structure, since the Danish area considered is divided into smaller market areas and wind power producers can choose different areas to bid their production Also, in our test case, not all wind farms are considered, and big offshore wind farms, such as Horns Rev, might change the level of aggregated forecast error for DK1. However, the righthand graph of Figure 4 shows that in $95 \%$ of the considered hours of our case study the following equality is a very good approximation of the true relationship that exists between forecast errors, spot price and imbalance prices:

$$
\sum_{x} \pi_{t+h}^{c,+}\left(d_{t+h}^{*}\right)_{+}+\pi_{t+h}^{c,-}\left(-d_{t+h}^{*}\right)_{+}=\pi_{t+h}^{c} \times \sum_{x} d_{t+h}^{*}
$$

where the sum is taken over all independent wind power producers $x$ of the system, and for any $z \in \mathbb{R},(z)_{+}$is zero if $z<0$ and $z$ otherwise. For the definition of the other terms, we refer to Subsection 2.3 describing the dayahead market model. The term on the left is the sum of imbalance costs over all market participants $\sum_{x} T_{t+h}^{c}$. It is the total amount of money that is paid or received by independent wind power producers for their imbalance. The right-hand side of Equation 6 is the aggregated forecast error (i.e. the wind power forecast error as seen by the TSO for the hourly resolution) multiplied by the spot price.

It should be noticed that Equation 6 is only a partial constraint for the imbalance prices, since having one equation for two unknowns leaves us a degree of freedom. Within the Danish system this degree of freedom is fixed if we assume that $\pi_{t+h}^{c,+}$ (respectively $\pi_{t+h}^{c,-}$ ) is null when $\sum_{x} d_{t+h}^{*}<0$ (respectively $\sum_{x} d_{t+h}^{*}>0$ ). This rule is indeed used by the Danish TSO, see e.g. [18]. In this case, Equation 6 makes it possible to obtain an explicit expression for $\pi_{t+h}^{c,-}$ and $\pi_{t+h}^{c,+}$ when these are not null:

$$
\pi_{t+h}^{c,-}=\pi_{t+h}^{c} \times \frac{\sum_{x} d_{t+h}^{*}}{\sum_{x \in I_{-}} d_{t+h}^{*}}, \quad \pi_{t+h}^{c,+}=\pi_{t+h}^{c} \times \frac{\sum_{x} d_{t+h}^{*}}{\sum_{x \in I_{+}} d_{t+h}^{*}},
$$

where $I_{+}$(resp. $I_{-}$) are the set of producers for which $d_{t+h}^{*}>0$ (resp. $\left.d_{t+h}^{*}<0\right)$. The two first graphs of Figure 4 suggest that in practice, these are inequalities more often than equalities (left equation being $\leq$ and right equation being $\geq$ ). In Equation 7, the left-hand side term measures the aggregation of errors in the considered area, and it will be close to one if the 
errors are all in the same direction, and close to zero if the errors compensate.
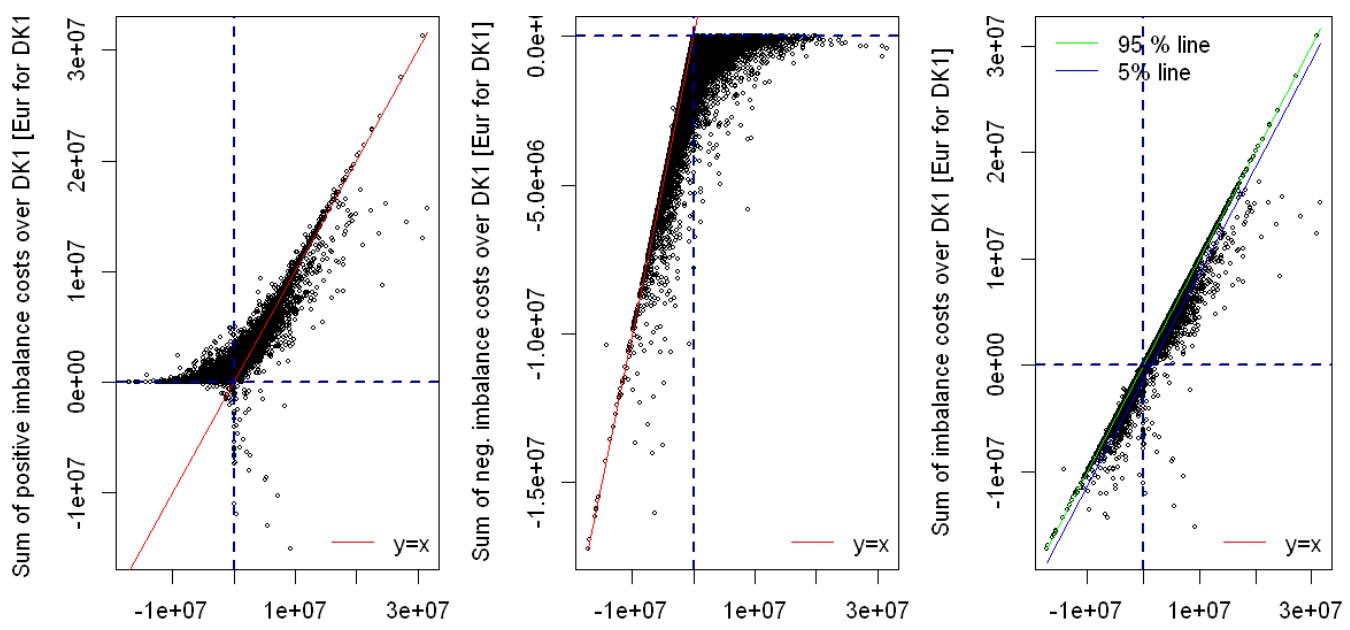

(Energy Forecast Errors of aggregated DK1* Spot price) [Eur]

Figure 4: Relationship between total imbalance costs (each point corresponds to the sum for the wind farms considered in DK1, at a fixed hour of the 682 considered days) and the price of the forecast error of the aggregation (i.e. energy forecast errors $\times$ spot price, again the total is for all considered wind farms). The first graph shows total positive imbalance costs (i.e. costs of positive imbalance), the second graph shows total negative imbalance costs and the third graph total imbalance costs.

\subsection{Future value of predictability as a design parameter}

Considering the benefits aggregation may have on predictability, it is clear that the Danish test case offers the moderate possibility of having a very high spatial smoothing effect, due to the correlation of the wind climate and predictability over the area of study. A greater smoothing effect may be obtained when considering a portfolio of wind farms across Europe or in larger countries with higher wind climate spatial variability, like Spain or France. In such larger areas, one can substantially improve predictability through aggregation, reducing the weight of imbalance costs appreciably. However, according to Equation 7, an increase in the smoothing effect of the errors at fixed spot prices would result in a substantial decrease in imbalance prices and hence imbalance costs. This would reduce the financial benefits of aggregation from the producer's point of view. In Addition, the financial gain from the producer's point of view of aggregating forecast errors in a 
European Wide market could also be decreased if transport capacity were to be remunerated. The results obtained in this paper could be different under a nodal price mechanism, such as the one studied in e.g. [19, 20].

The future increase of wind power penetration is also a factor that could modify imbalance prices, e.g. by increasing the average spot price or its volatility. However, Equation 7 shows that at a fixed level of aggregation, an increase in imbalance prices can only result from an increase in spot prices. It is clear that a simultaneous increase in spot prices and imbalance prices will not change the share of imbalance costs in wind power producers' revenue. Increased aggregation could only decrease the ratio between imbalance costs and base revenue.

One might argue that the Nordpool market is not a market that highly penalises imbalances. We have observed that in average the balancing prices are of the same order as the spot prices. This is illustrated in 4 and can be explained among others by the available flexibilities through strong in-

terconnections of Denmark with neighbouring countries or the existence of low-cost balancing power such as hydro in the Nordic countries. Higher balancing prices might be expected if these flexibilities would not be available. Similarly, balancing prices could be affected if wind power penetration in northern Europe would reach very high levels. It is however difficult to imagine that the balancing prices could, in average, exceed 10 times the average spot price. This would be a case where around $2 \%$ of revenue could be explained by predictability. Finally, it is likely that a factor of 10 would make storage profitable, which would result in reducing difference between balancing and spot prices.

\section{Conclusion}

In this paper, we have analyzed the financial benefit of increasing predictability from a producer's point of view. We based our analysis on a simplified market model with real-life wind power production and day-ahead market data from the Elspot market. We showed that the financial loss due to imbalance costs induced by imperfect predictions represents a low share of revenue in the day-ahead market. Only an increase in predictability resulting from aggregation could lead to a substantial increase in benefits. In this paper, we have quantified these potential gains through modeling the relationship that exists between total revenue, the capacity factor and predictability. The associated sensitivity analysis showed that in a case when aggregation 
is not considered, only $0.02 \%$ of the revenue's variance can be explained by predictability, while in a case where aggregation is considered, this proportion reaches $0.15 \%$. This low benefit from predictability is explained partly by the level of imbalance costs and partly by the strong positive correlation that exists between prediction errors and the capacity factor. Ultimately, this implies that in any resource assessment phase, lower predictability will go hand in hand with a high associated capacity factor. This makes predictability almost irrelevant in the resource assessment phase, at least from a wind power producer's point of view. Note that there is a difference between considering predictability in the resource assessment phase and considering predictability for a given installed wind farm. In the second case, not treated in this paper, the capacity factor is fixed and the role of predictability is much stronger.

Market imbalance cost reduction is not the only benefit a wind power producer can obtain from predictability. Within the O\& M cost breakdown, predictability can play a more important role, especially for offshore wind farms, where the lack of predictability leads not only to market imbalance costs but also to loss of availability due to downtime periods (turbines not accessible due to bad weather that was not well predicted in the maintenance strategy). Still, apart from specific rules in tenders for wind power installation projects, market imbalance costs constitute the only incentive for producers toward achieving more predictability.

While the results of this paper show the market's incentive action on wind power producers toward achieving greater predictability, it does not quantify the benefit of predictability from the system's point of view. Indeed, the effectiveness of the market measure does not necessarily coincide with the value of predictability with respect to the electric system, and might miss the benefits and costs brought about by longer-term investment. Further work should contain a systemic analysis in the spirit of the capacity value [22], in order to reveal the intrinsic value of increasing predictability for a given system at a given level of predictability.

This paper also provided a prospective analysis, based mainly on the relationship that exists between imbalance prices, spot prices and aggregation of prediction errors in the system. Under the assumption that the market mechanism will remain the same in the future, we have shown that the benefit of predictability for an independent producer participating in the electricity market is unlikely to increase.

We have shown that the obtained results would not be different with a 
larger spatial smoothing effect that could result from the consideration of a larger area. However, this result depends on the analyzed Nordpool market. Alternative market mechanisms, e.g. based on nodal pricing, would most likely give fundamentally different results. The analysis of such cases w.r.t. predictability makes part of the perspectives of this work.

\section{Acknowledgement}

The authors would like to thank the Danish TSO Energinet.dk for providing the wind farm data used in this study. We thank the European Commis-

sion for partially funding the FP7 project Safe wind (http:// www.safewind.eu) in the frame of which this work was performed.

[1] Pure Power - Wind energy targets for 2020 and 2030, A report by the European Wind Energy Association, Technical Report, EWEA, July 2011. Available online at www.ewea.org.

[2] A. Purvins, A. Zubaryeva, M. Llorente, E. Tzimas, A. Mercier, Challenges and options for a large wind power uptake by the european electricity system, Applied Energy 88 (2011) 1461 - 1469.

[3] G. Montes, E. Martín, Profitability of wind energy: short-term risk factors and possible improvements, Renewable and Sustainable Energy Reviews 11 (2007) 2191-2200.

[4] U. Focken, M. Lange, H. Waldl, Previento - a wind power prediction system with innovative upscaling algorithm., in: In Proc. Europ. Wind Energy Conf. EWEC, Copenhagen.

[5] I. Marti, et al, What performance can be expected by short-term wind power prediction models depending on site characteristics?, in: In CDRom Proceedings, European Wind Energy Conference EWEC.

[6] P. Pinson, Estimation of the uncertainty in wind power forecasting, Ph.D. thesis, Ecole des Mines de Paris, Center for Energy and Processes, Rue Claude Daunesse, Sophia Antipolis, 2006.

[7] P. Pinson, C. Chevallier, G. Kariniotakis, Trading wind generation from short-term probabilistic forecasts of wind power, IEEE Transactions on Power Systems 22 (2007). 
[8] F. Bourry, Management of uncertainties related to renewable generation participation in electricity market, Ph.D. thesis, Ecole des Mines de Paris, Center for Energy and Processes, Rue Claude Daunesse, Sophia Antipolis, 2009.

[9] V. Marano, G. Rizzo, F. A. Tiano, Application of dynamic programming to the optimal management of a hybrid power plant with wind turbines, photovoltaic panels and compressed air energy storage, Applied Energy (2012) - .

[10] J. Morales, A. Conejo, J. Perez-Ruiz, Short-term trading for a wind power producer, Power Systems, IEEE Transactions on 25 (2010) 554 -564 .

[11] O. Grothe, J. Schnieders, Spatial dependence in wind and optimal wind power allocation: A copula-based analysis, Energy Policy 39 (2011) $4742-4754$.

[12] J. Lin, Y. zhang Sun, L. Cheng, W. zhong Gao, Assessment of the power reduction of wind farms under extreme wind condition by a high resolution simulation model, Applied Energy (2011) -.

[13] D. Pudjianto, C. Ramsay, G. Strbac, Virtual power plant and system integration of distributed energy resources, Renewable Power Generation, IET 1 (2007) $10-16$.

[14] L. Frias, U. Irigoyen, E. Pascal, E. Cantero, Y. Loureiro, P. Correia, S. Lozano, J. Sanz, Functional data analysis applied to the problem of wind farm aggregation, in: Proc. of the European Wind Energy Conference, Brussels, Belgium.

[15] A. Saltelli, S. Tarantola, On the relative importance of input factors in mathematical models, Journal of the American Statistical Association 97 (2002) 702-709.

[16] A. Liaw, M. Wiener, Classification and regression by randomforest, $\mathrm{R}$ News 2 (2002) 18-22.

[17] R Development Core Team, R: A Language and Environment for Statistical Computing, R Foundation for Statistical Computing, Vienna, Austria, 2011. ISBN 3-900051-07-0. 
[18] J. Matevosyan, L. Soder, Minimization of imbalance cost trading wind power on the short-term power market, Power Systems, IEEE Transactions on 21 (2006) $1396-1404$.

[19] L. Baringo, A. Conejo, Wind power investment within a market environment, Applied Energy 88 (2011) 3239 - 3247.

[20] C. Ruiz, A. Conejo, Pool strategy of a producer with endogenous formation of locational marginal prices, Power Systems, IEEE Transactions on 24 (2009) $1855-1866$.

[21] A. Tindal, K. Harman, C. Johnson, A. Schwarz, A. Garrad,G. Hassan Validation of the GH energy and uncertainty predictions by comparison to actual production In Proc. of the AWEA Wind Resource and Project Energy Assessment Workshop (2007)

[22] A. Keane, M. Milligan, C. Dent, B. Hasche, C. D'Annunzio, K. Dragoon, H. Holttinen, N. Samaan, L. Soder, M. O'Malley, Capacity value of wind power, IEEE Transactions on Power Systems 26 (2011) 564 -572. 